BMJ Open Diabetes Research \& Care

\title{
Long-term follow-up of a randomized clinical trial comparing glycemic excursion minimization (GEM) to weight loss (WL) in the management of type 2 diabetes
}

\author{
Daniel J Cox (D) ,' Tamara Oser, ${ }^{2}$ Matthew Moncrief, ${ }^{3}$ Mark Conaway, ${ }^{4}$ \\ Anthony McCall ${ }^{5}$
}

To cite: Cox DJ, Oser T, Moncrief $\mathrm{M}$, et al. Long-term follow-up of a randomized clinical trial comparing glycemic excursion minimization (GEM) to weight loss (WL) in the management of type 2 diabetes. BMJ Open Diab Res Care 2021;9:e002403. doi:10.1136/ bmjdrc-2021-002403

Received 21 May 2021 Accepted 17 October 2021

Check for updates

\section{(c) Author(s) (or their} employer(s)) 2021. Re-use permitted under CC BY-NC. No commercial re-use. See rights and permissions. Published by BMJ.

\section{${ }^{1}$ Psychiatry, University of} Virginia, Charlottesville, Virginia, USA

${ }^{2}$ Family Medicine, University of Colorado Anschutz Medical Campus, Aurora, Colorado, USA ${ }^{3}$ Center for Behavioral Medicine Research, University of Virginia School of Medicine, Charlottesville, Virginia, USA ${ }^{4}$ Department of Public Health Sciences, University of Virginia School of Medicine, Charlottesville, Virginia, USA ${ }^{5}$ Medicine, University of Virginia, Charlottesville, Virginia, USA

\section{Correspondence to} Dr Daniel J Cox; djc4f@hscmail.mcc.virginia. edu

\section{ABSTRACT}

Introduction We previously reported the physical, psychological and behavioral 3-month post-treatment results of a randomized controlled trial comparing glycemic excursion minimization (GEM) versus conventional weight loss (WL) therapy in the management of type 2 diabetes (T2D). GEM is a paradigm shift in the lifestyle management of T2D that focuses on reducing postnutrient glucose excursions, rather than reducing weight. We now present the 13-month follow-up results.

Research design and methods The initial study sample of 172 were $30-80$ years old, had T2D for $\leq 10$ years, an $\mathrm{HbA} 1 \mathrm{C}$ $\geq 6.8 \%$ ( $51 \mathrm{mmol} / \mathrm{mol}$ ), and were not using insulin. Participants were randomized to 6 hours of group treatment, either to WL or one of three versions of GEM. GEM groups differed in degree of blood glucose (BG) feedback provided during treatment: no recommended feedback, systematic capillary BG feedback before and after nutrient intake and physical activity, or continuous glucose monitoring. Since these GEM groups did not differ in pre-post improvement they were combined for initial and current analyses. Of those who completed the 3-month postassessment, $100 \%$ and $96 \%$ of the WL and GEM participants completed the 13-month follow-up assessment. Results Pre to follow-up within-group comparisons indicated WL participants sustained improvement in body mass index (BMI) $(-0.9 \pm 1.4, p=0.001)$. GEM participants continued to benefit in their HbA1c $(-0.5 \pm 1.4, p<0.001), B M I(-1 \pm 1$, $p<0.001)$, high-density lipoprotein $(p<0.001)$, reduction of carbohydrate ingestion $(p<0.001)$, self-monitoring of blood glucose satisfaction $(p<0.001)$ and frequency $(p<0.001)$, diabetes knowledge $(p<0.001)$, diabetes empowerment $(p<0.001)$, and both diabetes distress emotional $(p=0.009)$ and regimen $(p=0.001)$ subscales. Forty-two percent and $52 \%$ of WL and GEM participants, respectively, were classified as responders (individuals whose A1c dropped by at least $-0.5 \%$ ), with a mean $\mathrm{HbA} 1 \mathrm{c}$ reduction of $-1.2 \%$ and $-1.5 \%$. Neither WL nor GEM responders differed from non-responders in baseline demographics, psychological or disease severity variables. While WL responders could not be predicted, $73 \%$ of GEM responders were predicted by post minus pretreatment reductions of $\mathrm{HbA1c}$, diabetes medication and $\mathrm{BMI}$.

Conclusions While WL sustained improvement in BMI, GEM sustained benefits across a broad range of physical, behavioral and psychological parameters, beneficial for clinicians and

\section{Significance of this study}

What is already known about this subject?

- The efficacy of glycemic excursion minimization (GEM) in the short term is equal to or greater than conventional weight loss $(\mathrm{WL})$ therapy in improving primary $(\mathrm{HbA1c})$ and secondary (cardiovascular risk, diabetes empowerment, diabetes distress, depressive symptoms, highdensity lipoprotein (HDL)) outcomes.

What are the new findings?

- This is the first long-term follow-up of an alternative type 2 diabetes lifestyle intervention that focuses on minimizing postnutrient glucose excursions: GEM.

- This new approach demonstrated sustained benefits in $\mathrm{HbA1c}, \mathrm{HDL}$, body mass index (BMI), diabetes empowerment, diabetes distress, and blood glucose monitoring, over 13 months despite having no maintenance intervention.

> Benefits of these two lifestyle interventions (GEM and conventional WL) were unrelated to baseline measures of demographics, psychological function or disease severity.

- Being a long-term GEM responder was predicted by short-term reduction of $\mathrm{HbA1C}, \mathrm{BMI}$ and the use of diabetic medications.

How might these results change the focus of research or clinical practice?

> These findings suggest that GEM is a brief, sustainable and effective lifestyle treatment option for adults with type 2 diabetes that may be especially relevant to patients who do not need or are unable to achieve long-term WL; GEM may help primary care providers who manage most patients with type 2 diabetes. Future research needs to focus on increasing the proportion of long-term responders, possibly by boosting short-term benefits.

adults with T2D. This may be especially relevant for primary care physicians who manage about $90 \%$ of patients with T2D. Trial registration number NCT03196895. 


\section{INTRODUCTION}

Unlike pharmacological interventions for type 2 diabetes (T2D) that reflect a variety of mechanisms, lifestyle interventions are primarily limited to a single focus on weight loss (WL). However, some adults with T2D do not need to lose weight (approximately 15\%), ${ }^{1}$ do not want to lose weight or cannot lose weight and keep it off for the duration of T2D. ${ }^{2}$ Consequently, we developed a paradigm shift in lifestyle intervention that reduces postnutrient blood glucose (BG) excursions, glycemic excursion minimization (GEM). GEM reduces BG area under the curve by identifying a person's food choices that minimize BG elevations and physical activities that hasten BG recovery, empowering patients to make choices that diminish BG excursions and thus reduce HbAlc and cardiovascular risk. ${ }^{34}$ GEM has been found to be superior to routine care. ${ }^{5}{ }^{6}$ Most recently, we compared WL to GEM at a 3-month follow-up ${ }^{7}$ and found while WL reduced body mass index (BMI) ( $p=0.005)$, GEM demonstrated a significant reduction in HbA1c $(\mathrm{p}=0.005)$, BMI $(\mathrm{p}=0.013)$, carbohydrate intake $(p=0.001), B G$ response to a glucose challenge $(p=0.02)$, and cardiovascular risk $(p=0.003)$. Only GEM participants significantly improved diabetes empowerment, diabetes distress, depressive symptoms, steps/day, and reduced calories/day. Neither intervention worsened lipids.

A critical aspect for general use requires sustainability, that is, continued benefit from pretreatment, which is needed to determine its potential as a pragmatic intervention, but this had not been investigated.$^{5-8}$ Therefore, we present the first long-term follow-up of GEM, testing the hypothesis that GEM will be equivalent or superior to WL in sustaining its benefits in the primary (HbAlc) and secondary variables (physical, psychological, and behavioral parameters) found improved at a 3-month follow-up, without any maintenance intervention.

\section{RESEARCH DESIGN AND METHODS}

After signing a University of Virginia Health Sciences Center Institutional Review Board approved consent form, participants completed a baseline assessment and 6 hours of group training. This was followed by a 3-month postassessment and now a 13-month follow-up. Thirty-six WL and 123 GEM adults completed the postassessment and $100 \%$ and $97 \%$ of these groups completed the 13-month follow-up assessment, respectively. One participant was deceased, one moved out of town, one refused to come in due to COVID-19, and one could never be contacted in the GEM groups. There were no adverse events reported in either group during follow-up.

\section{Assessments}

All assessments included blood tests (HbA1c and lipids), quantification of cardiovascular risk (UK Prospective Diabetes Study Outcomes Model 2), ${ }^{9}$ incorporating HbA1c, systolic blood pressure, total cholesterol, highdensity lipoprotein (HDL) cholesterol, smoking, age, sex, diabetes duration, ethnicity and presence of atrial fibrillation, psychological functioning (diabetes distress (emotional and regimen subscales) $),{ }^{10}$ diabetes empowerment, ${ }^{11}$ Patient Health Questionnaire-9 (PHQ-9) to assess depressive symptoms, ${ }^{12}$ and the Glucose Monitor Satisfaction Survey. ${ }^{13}$ Participants' frequency of selfmonitoring of blood glucose (SMBG) over the previous month was extracted from their BG meter's history function. The Medication Effect Score (MES) was also calculated. ${ }^{14}$ MES is a metric that converts a patient's doses and types of BG-lowering medications into a summed common metric-its average HbAlc-lowering potential. Change in HbAlc and MES were combined into the total treatment effect (TTE) to identify the unique contribution of the lifestyle intervention. ${ }^{5}$ The TTE is quantified as the change in HbAlc minus the change in MES.

\section{Training}

All participants were given a training manual to read, food diaries to complete with their training sessions, activity monitors, and meters and strips for SMBG. Provision of SMBG supplies was discontinued after the 3-month postassessment.

GEM training involved four 90 min group sessions focused on reducing postnutrient glucose excursions (area under the curve) by the mechanisms of: (1) educating participants on the glycemic impact of different foods and activity choices, (2) diminishing BG elevations through moderate reduction of certain carbohydrates, and (3) hastening BG recovery by increasing postprandial and routine physical activity. There were three subgroups of GEM with varied BG feedback, but these groups were merged for analyses in this and the original short-term presentation ${ }^{7}$ because they did not differ from one another in primary or secondary outcome measures. WL training involved six 60 min group sessions adapted from lessons in the Centers for Disease Control and Prevention's (CDC) 'Prevent T2' curriculum aimed at: (1) reducing caloric intake and (2) increasing moderate to vigorous physical activity. ${ }^{15}$ No maintenance program was employed between postassessment and 13-month follow-up for either GEM or WL. For more details, see our original publication. ${ }^{7}$

\section{Statistical methods}

The analyses included all available participants for each group and were like those done for the 3-month postassessment report. ${ }^{7}$ An analysis of covariance of pre to follow-up change scores, covarying baseline metrics, was conducted in SAS V.9.4. Orthogonal contrasts compared GEM to WL. The Benjamini-Hochberg procedure ${ }^{16}$ was employed to control for multiple comparisons, correcting for all $p$ values in each table. $P$ value levels with an asterisk (*) in tables indicate significant contrasts following this correction. Logistic regression analysis was employed to identify predictors of sustainability. Only those variables significant at post-treatment were entered into the regression model to predict responders at follow-up. 
Table 1 Changes from baseline to 13 months for the WL and GEM groups, and statistical significance for changes from baseline, both within and between groups

\begin{tabular}{|c|c|c|c|c|c|}
\hline \multirow[b]{2}{*}{ Variables } & \multicolumn{2}{|c|}{$\mathrm{M}(\mathrm{SD})$ baseline to 13 months $\Delta$} & \multicolumn{3}{|c|}{$P$ value level } \\
\hline & WL & GEM & $\begin{array}{l}\text { WL versus } \\
\text { GEM }\end{array}$ & WL & GEM \\
\hline Sample size & 36 & 123 & & & \\
\hline \multicolumn{6}{|l|}{ Primary outcome variables } \\
\hline $\mathrm{HbA} 1 \mathrm{c}(\%$ and $(\mathrm{mmol} / \mathrm{mol}))$ & $\begin{array}{l}-0.2 \pm 1.3 \\
(-2.2 \pm 14.2)\end{array}$ & $\begin{array}{l}-0.5 \pm 1.4 \\
(-5.5 \pm 15.3)\end{array}$ & 0.33 & 0.33 & $<0.001^{*}$ \\
\hline \multicolumn{6}{|l|}{ Secondary outcome variables } \\
\hline \% cardiovascular risk (UKPDS-OM2) & $0.6 \pm 4.7$ & $-0.7 \pm 6.5$ & 0.21 & 0.47 & 0.26 \\
\hline Diabetes empowerment & $-1.3 \pm 6.0$ & $2.2 \pm 5.7$ & $0.005^{\star}$ & 0.28 & $<0.001^{*}$ \\
\hline Diabetes distress (emotional) & $0.1 \pm 0.9$ & $-0.3 \pm 1.0$ & 0.21 & 0.76 & $0.009^{*}$ \\
\hline Diabetes distress (regimen) & $-0.4 \pm 1.0$ & $-0.4 \pm 1.3$ & 0.93 & 0.03 & $0.001^{*}$ \\
\hline Depression (Patient Health Questionnaire-9) & $-0.2 \pm 4.2$ & $-0.4 \pm 3.9$ & 0.60 & 0.79 & 0.22 \\
\hline \multicolumn{6}{|l|}{ Side effect variables } \\
\hline $\mathrm{LDL}(\mathrm{mmol} / \mathrm{L})$ & $0 \pm 0.6346$ & $-0.0647 \pm 0.8012$ & 0.65 & 1.00 & 0.401 \\
\hline $\mathrm{HDL}(\mathrm{mmol} / \mathrm{L})$ & $0.0345 \pm 0.1579$ & $0.0723 \pm 0.1726$ & 0.24 & 0.20 & $<0.001^{*}$ \\
\hline Triglycerides (mmol/L) & $0.1581 \pm 0.5269$ & $-0.0761 \pm 1.593$ & 0.89 & 0.08 & 0.61 \\
\hline Total cholesterol (mmol/L) & $0.1092 \pm 0.6419$ & $0.0011 \pm 0.9714$ & 0.73 & 0.31 & 0.99 \\
\hline \multicolumn{6}{|l|}{ Mechanism variables } \\
\hline Diabetes knowledge & $0.3 \pm 2.6$ & $2.5 \pm 2.7$ & $<0.001^{\star}$ & 0.57 & $<0.001^{*}$ \\
\hline Body mass index (BMI) & $-0.9 \pm 1.4$ & $-1 \pm 1.6$ & 0.56 & $0.001^{*}$ & $<0.001^{*}$ \\
\hline $\begin{array}{l}\text { Carbohydrates Routinely Consumed } \\
\text { (servings) }\end{array}$ & $-1.8 \pm 16.3$ & $-13.3 \pm 19$ & $<0.01^{*}$ & 0.57 & $<0.001^{*}$ \\
\hline SMBG readings (30 days) & $2.4 \pm 22.5$ & $12.6 \pm 31.8$ & 0.18 & 0.53 & $<0.001^{*}$ \\
\hline Glucose Monitor Satisfaction Survey (GMSS) & $0.9 \pm 0.6$ & $0.3 \pm 0.5$ & 0.04 & 0.44 & $<0.001^{*}$ \\
\hline Medication Effect Score (MES) & $0.1 \pm 0.5$ & $0 \pm 0.6$ & 0.24 & 0.11 & 0.44 \\
\hline
\end{tabular}

${ }^{*} P$ value levels were calculated with a false discovery rate of 0.05 .

GEM, glycemic excursion minimization; HDL, high-density lipoprotein; LDL, low-density lipoprotein; SMBG, self-monitoring of blood glucose; UKPDS-OM2, UK Prospective Diabetes Study Outcomes Model 2; WL, weight loss.

\section{RESULTS}

Table 1 displays the means and SDs of follow-up change scores (13 months minus baseline) for the outcome variables.

\section{Primary outcome variables}

Within-group analyses indicated only the GEM participants significantly sustained improvement in HbAlc $(-0.5 \%, \mathrm{p}<0.001)$. Between-group analyses indicated no differences between groups (table 1).

\section{Secondary outcome variables}

As shown in table 1, between-group analyses indicated, compared with WL, GEM had greater sustained benefits regarding diabetes empowerment $(\mathrm{p}=0.005)$.

Within-group analyses indicated WL had no sustained secondary benefits. GEM participants showed sustained improvements in diabetes empowerment $(\mathrm{p}<0.001)$ and diabetes distress (emotional $(\mathrm{p}=0.009)$ and regimen $(\mathrm{p}=0.001))$.

\section{Side effect variables}

Neither treatment worsened lipids, but within-group analysis indicated GEM significantly sustained improvement of HDL $(\mathrm{p}<0.001$, table 1$)$.

\section{Mechanism variables}

Between-group analysis indicated GEM sustained more improvement in diabetes knowledge $(p<0.001)$ and reduction in carbohydrate intake $(\mathrm{p}<0.001$, table 1$)$.

Within-group analyses indicated both $\mathrm{WL}$ and GEM lowered BMI ( $p=0.001$ and $p<0.001$, respectively) at 13 months. Only GEM significantly improved diabetes knowledge $(\mathrm{p}<0.001)$, reduced carbohydrate intake $(p<0.001)$, increased frequency of SMBG $(p<0.001)$, and improved satisfaction with SMBG $(\mathrm{p}<0.001)$.

\section{Post hoc analyses}

Responders versus non-responders

Table 2 presents changes over 13 months (13-month follow-up minus baseline) comparing responders to 
Table 2 Mean and SD of the change from baseline to 13 months for responders and non-responders in the WL and GEM groups, with contrasts ( $p$ value levels)

\begin{tabular}{|c|c|c|c|c|c|c|}
\hline & \multicolumn{3}{|c|}{ WL pre to 13 months $\Delta$} & \multicolumn{3}{|c|}{ GEM pre to 13 months $\Delta$} \\
\hline & Responders & Non-responders & $P$ value & Responders & $\begin{array}{l}\text { Non- } \\
\text { responders }\end{array}$ & $P$ value \\
\hline \multicolumn{7}{|l|}{ Primary outcome variable } \\
\hline $\mathrm{HbA} 1 \mathrm{c}(\%$ and $(\mathrm{mmol} / \mathrm{mol}))$ & $\begin{array}{l}-1.2 \pm 0.7 \\
(-13.1 \pm 7.7)\end{array}$ & $\begin{array}{l}0.5 \pm 1.2 \\
(5.5 \pm 13.1)\end{array}$ & $<0.001^{\star}$ & $\begin{array}{l}-1.5 \pm 1.0 \\
(-16.4 \pm 10.9)\end{array}$ & $\begin{array}{l}0.5 \pm 1.0 \\
(5.5 \pm 10.9)\end{array}$ & $<0.001^{*}$ \\
\hline Total treatment effect (TTE) & $-1.2 \pm 0.7$ & $0.8 \pm 1.1$ & $<0.001^{*}$ & $-1.6 \pm 1.0$ & $0.8 \pm 1.0$ & $<0.001^{*}$ \\
\hline \multicolumn{7}{|l|}{ Secondary outcome variables } \\
\hline $\begin{array}{l}\text { \% cardiovascular risk } \\
\text { (UKPDS-OM2) }\end{array}$ & $-2.2 \pm 4.8$ & $2.5 \pm 3.7$ & $<0.01^{*}$ & $-2.8 \pm 5.3$ & $1.6 \pm 7.0$ & $<0.001^{*}$ \\
\hline Diabetes empowerment & $0.8 \pm 5.6$ & $-2.8 \pm 6.0$ & 0.12 & $3.6 \pm 4.8$ & $0.7 \pm 6.3$ & $0.007^{*}$ \\
\hline Diabetes distress (emotional) & $0.0 \pm 0.9$ & $0.1 \pm 0.9$ & 0.80 & $-0.5 \pm 0.9$ & $0.0 \pm 1.1$ & $0.019^{*}$ \\
\hline Diabetes distress (regimen) & $-0.3 \pm 1.2$ & $-0.5 \pm 0.8$ & 0.59 & $-0.7 \pm 1.3$ & $-0.1 \pm 1.2$ & $0.007^{*}$ \\
\hline $\begin{array}{l}\text { Depression (Patient Health } \\
\text { Questionnaire-9) }\end{array}$ & $1.3 \pm 3.7$ & $-1.3 \pm 4.3$ & 0.11 & $-1.0 \pm 3.2$ & $0.1 \pm 4.4$ & 0.14 \\
\hline $\begin{array}{l}\text { Medication Effect Score } \\
\text { (MES) }\end{array}$ & $0.0 \pm 0.3$ & $0.3 \pm 0.6$ & 0.09 & $-0.2 \pm 0.5$ & $0.3 \pm 0.6$ & $<0.001^{*}$ \\
\hline \multicolumn{7}{|l|}{ Side effect variables } \\
\hline LDL (mmol/L) & $-0.0665 \pm 0.4619$ & $0.0443 \pm 0.7354$ & 0.62 & $-0.074 \pm 0.7421$ & $-0.0538 \pm 0.8723$ & 0.90 \\
\hline $\mathrm{HDL}(\mathrm{mmol} / \mathrm{L})$ & $0.0776 \pm 0.144$ & $0.0037 \pm 0.1634$ & 0.17 & $0.1204 \pm 0.1785$ & $0.0199 \pm 0.1506$ & $0.001^{*}$ \\
\hline Triglycerides (mmol/L) & $0.324 \pm 0.542$ & $0.2478 \pm 0.5099$ & 0.23 & $-0.0611 \pm 0.894$ & $-0.0925 \pm 2.1165$ & 0.92 \\
\hline Total cholesterol (mmol/L) & $0.638 \pm 0.509$ & $0.1416 \pm 0.7326$ & 0.73 & $0.0309 \pm 0.8414$ & $-0.0314 \pm 1.1026$ & 0.73 \\
\hline \multicolumn{7}{|l|}{ Mechanism variables } \\
\hline Diabetes knowledge & $0.3 \pm 2.8$ & $0.3 \pm 2.6$ & 0.95 & $2.8 \pm 2.8$ & $2.2 \pm 2.6$ & 0.24 \\
\hline Body mass index (BMI) & $-1.3 \pm 1.3$ & $-0.5 \pm 1.4$ & 0.08 & $-1.5 \pm 1.7$ & $-0.5 \pm 1.4$ & $0.002^{*}$ \\
\hline $\begin{array}{l}\text { Carbohydrates Routinely } \\
\text { Consumed (servings) }\end{array}$ & $-2.7 \pm 16.7$ & $1.2 \pm 16.5$ & 0.82 & $-17.2 \pm 16.4$ & $-8.7 \pm 21.5$ & $0.021^{*}$ \\
\hline SMBG readings (30 days) & $1.6 \pm 22.8$ & $3.0 \pm 23.0$ & 0.86 & $13.1 \pm 30.0$ & $12.1 \pm 34.0$ & 0.87 \\
\hline $\begin{array}{l}\text { Glucose Monitor Satisfaction } \\
\text { Survey (GMSS) }\end{array}$ & $0.2 \pm 0.6$ & $0.0 \pm 0.6$ & 0.31 & $0.4 \pm 0.6$ & $0.1 \pm 0.5$ & $0.007^{\star}$ \\
\hline
\end{tabular}

*Significant with a false discovery rate of $0.05 .{ }^{16}$

GEM, glycemic excursion minimization; HDL, high-density lipoprotein; LDL, low-density lipoprotein; SMBG, self-monitoring of blood glucose; UKPDS-OM2, UK Prospective Diabetes Study Outcomes Model 2; WL, weight loss.

non-responders for both treatments. Responders were defined as any participant who reduced TTE by -0.5 or more. The TTE is a more discriminating metric than HbAlc with regard to comparing treatment efficacy, as change in A1c does not consider any concurrent change in BG-lowering medications prescribed over follow-up.

\section{Primary outcome variables}

Fifty-two percent of GEM participants and $42 \%$ of WL participants were classified as responders, and the mean reduction in TTE was -1.6 and -1.2 , respectively $(\mathrm{p}=0.23)$. GEM responders reduced HbA1c by $1.5 \%$ ( $16.4 \mathrm{mmol} /$ $\mathrm{mol})$ and WL responders by $1.2 \%(13.1 \mathrm{mmol} / \mathrm{mol})$ $(\mathrm{p}=0.27)$.

\section{Secondary outcome variables}

At 13-month follow-up, within-group analyses indicated both WL and GEM responders, compared with non-responders, sustained reduction in reduced cardiovascular risk (respectively $\mathrm{p}=0.003$ and $\mathrm{p}<0.001$ ). GEM responders, compared with GEM non-responders, additionally sustained improvement in the same variables as all GEM participants in table 1 , but also were taking significantly less diabetes medication at follow-up $(\mathrm{p}<0.001)$.

\section{Side effect variables}

Differences comparing responders to non-responders were similar to those in table 1 .

\section{Mechanism variables}

WL responders, compared with non-responders, did not differ on any mechanism variables. GEM responders, compared with non-responders, demonstrated a greater reduction in BMI $(\mathrm{p}=0.002)$ and carbohydrate ingestion $(\mathrm{p}=0.021)$, and greater satisfaction with SMBG $(\mathrm{p}=0.007)$. 
Table 3 Baseline variables for responders and non-responders in the GEM and WL groups, with contrasts ( $p$ value levels)

\begin{tabular}{|c|c|c|c|c|c|c|c|}
\hline \multirow[b]{2}{*}{ Baseline variable } & \multirow[b]{2}{*}{ Test } & \multicolumn{3}{|l|}{ WL } & \multicolumn{3}{|l|}{ GEM } \\
\hline & & Responders & $\begin{array}{l}\text { Non- } \\
\text { responders }\end{array}$ & $\begin{array}{l}P \\
\text { value }\end{array}$ & Responders & $\begin{array}{l}\text { Non- } \\
\text { responders }\end{array}$ & $\begin{array}{l}P \\
\text { value }\end{array}$ \\
\hline Demographic variable & & 15 & 21 & NA & 61 & 57 & NA \\
\hline Age (years) & t-test & $56.9 \pm 12.8$ & $59.3 \pm 9.5$ & 0.52 & $58.1 \pm 12.3$ & $54.7 \pm 10.7$ & 0.11 \\
\hline Education (years) & t-test & $15.2 \pm 2.9$ & $15.4 \pm 2.5$ & 0.84 & $16.1 \pm 3.0$ & $16.1 \pm 3.2$ & 0.99 \\
\hline Gender (\% female) & $X^{2}$ & $40 \%$ & $67 \%$ & 0.11 & $53 \%$ & $68 \%$ & 0.08 \\
\hline Race (\% white) & $X^{2}$ & $80 \%$ & $67 \%$ & 0.12 & $82 \%$ & $72 \%$ & 0.13 \\
\hline Body mass index (BMI) & t-test & $34.4 \pm 7.9$ & $35.2 \pm 6.6$ & 0.74 & $33.8 \pm 6.3$ & $35.7 \pm 5.6$ & 0.09 \\
\hline \multicolumn{8}{|l|}{ Psychological variables } \\
\hline Diabetes empowerment & t-test & $29.3 \pm 3.7$ & $31.6 \pm 3.6$ & 0.09 & $30.0 \pm 4.1$ & $30.0 \pm 5.2$ & 0.99 \\
\hline Diabetes distress (emotional) & t-test & $2.1 \pm 0.9$ & $2.4 \pm 1.1$ & 0.43 & $2.2 \pm 1.1$ & $2.6 \pm 1.1$ & 0.06 \\
\hline Diabetes distress (regimen) & t-test & $2.9 \pm 0.9$ & $3.3 \pm 1.3$ & 0.34 & $2.9 \pm 1.3$ & $3.2 \pm 1.4$ & 0.23 \\
\hline $\begin{array}{l}\text { Depression (Patient Health } \\
\text { Questionnaire-9) }\end{array}$ & t-test & $5.1 \pm 4.6$ & $4.1 \pm 4.0$ & 0.51 & $3.7 \pm 4.2$ & $4.8 \pm 5.1$ & 0.21 \\
\hline \multicolumn{8}{|l|}{ Disease severity } \\
\hline $\mathrm{HbA} 1 \mathrm{c}(\%$ and $(\mathrm{mmol} / \mathrm{mol}))$ & t-test & $8.4 \pm 1.0(68.6 \pm 11.1)$ & $\begin{array}{l}8.1 \pm 1.0 \\
(65.1 \pm 10.6)\end{array}$ & 0.34 & $\begin{array}{l}8.4 \pm 1.5 \\
(68.6 \pm 16.3)\end{array}$ & $\begin{array}{l}8.3 \pm 1.2 \\
(67.3 \pm 13)\end{array}$ & 0.62 \\
\hline Medication Effect Score & t-test & $1.2 \pm 0.8$ & $1.0 \pm 0.7$ & 0.39 & $1.2 \pm 0.8$ & $1.1 \pm 0.7$ & 0.33 \\
\hline Years with diabetes & t-test & $4.8 \pm 3.0$ & $6.0 \pm 2.0$ & 0.26 & $5.5 \pm 3.1$ & $5.4 \pm 3.3$ & 0.87 \\
\hline
\end{tabular}

GEM, glycemic excursion minimization; NA, not applicable; WL, weight loss.

\section{Covariates of responsiveness}

Table 3 presents baseline demographic, psychological and disease variables. These did not differentiate responders from non-responders for either intervention, including the demographic variables of age, level of education, sex, race, BMI; psychological variables of diabetes empowerment and distress, and depression; or the disease severity variables of HbAlc, MES and duration of disease.

Table 4 displays short-term improvements (3-month postassessment minus baseline) for 13-month responders versus non-responders. Short-term improvements in WL participants did not differentiate long-term responders from non-responders. However, compared with GEM non-responders, GEM responders had significantly greater short-term improvement regarding HbAlc $(\mathrm{p}<0.001)$, cardiovascular risk $(\mathrm{p}=0.008)$, diabetes distress emotional $(\mathrm{p}<0.015)$ and regimen $(\mathrm{p}<0.001)$ subscales, HDL $(\mathrm{p}<0.01)$, BMI $(\mathrm{p}<0.001)$, carbohydrate ingestion $(p<0.012)$, SMBG frequency $(p<0.022)$, satisfaction with SMBG $(\mathrm{p}<0.009)$, and MES $(\mathrm{p}<0.015)$.

Incorporating short-term improvements to predict sustainability, a stepwise logistic regression was performed to predict responders. Since there were no documented short-term benefits that differentiated WL responders, such a regression could not be performed. Logistic regression correctly classified $73 \%$ of GEM responders and $78 \%$ of GEM non-responders based on short-term improvements in A1c, BMI and MES $(\mathrm{p}<0.001)$ (see table 5).

\section{CONCLUSIONS}

This study set out to investigate whether GEM was an effective alternative lifestyle intervention to $\mathrm{WL}$ for adults with T2D, giving clinicians and patients a new treatment option. Between-group analyses demonstrated GEM was equivalent or superior to $\mathrm{WL}$ at a 13-month follow-up. Compared with WL, GEM demonstrated greater improvement in empowerment, diabetes knowledge as related to GEM principles, reduction in carbohydrate consumption, and marginal $(\mathrm{p}=0.04)$ improvement in satisfaction with BG monitoring. These differences could be anticipated since GEM is an empowerment program, ${ }^{17}{ }^{18}$ designed to provide information about the impact of carbohydrates and physical activity on postnutrient BG excursions through BG feedback, allowing patients to make choices to reduce postnutrient glucose excursions. When considering within-group analyses, WL achieved its objective to sustain BMI, while GEM did this along with improved HbA1c, HDL, frequency of and satisfaction with SMBG, diabetes empowerment and diabetes distress.

From a clinician's perspective, it may be more important to know how many benefit from an intervention rather than the intervention mean group improvements. Consequently, we investigated how many participants demonstrated a meaningful reduction in HbAlc, that is, a reduction of at least $0.5 \%$. Again, GEM was equivalent to WL, with $52 \%$ vs $42 \%$ qualifying as responders, respectively. Within-group analyses demonstrated, compared with WL non-responders, WL responders had 
Table 4 Mean and SD of the change from baseline to 3 months for responders and non-responders in the WL and GEM groups, with contrasts ( $p$ value levels)

\begin{tabular}{|c|c|c|c|c|c|c|}
\hline & \multicolumn{3}{|l|}{ WL pre to post $\Delta$} & \multicolumn{3}{|c|}{ GEM pre to post $\Delta$} \\
\hline & Responders & Non-responders & $\begin{array}{l}P \\
\text { value }\end{array}$ & Responders & Non-responders & $P$ value \\
\hline \multicolumn{7}{|l|}{ Primary outcome variables } \\
\hline $\mathrm{HbA} 1 \mathrm{c}(\%$ and $(\mathrm{mmol} / \mathrm{mol}))$ & $\begin{array}{l}-0.7 \pm 1.4 \\
(-7.7 \pm 15.3)\end{array}$ & $0.0 \pm 1.2(0 \pm 13.1)$ & 0.13 & $\begin{array}{l}-1.5 \pm 1.2 \\
(-16.4 \pm 13.1)\end{array}$ & $\begin{array}{l}-0.05 \pm 0.9 \\
(-0.5 \pm 9.8)\end{array}$ & $<0.001^{*}$ \\
\hline Total treatment effect (TTE) & $-0.8 \pm 1.5$ & $0.2 \pm 1.6$ & 0.05 & $-1.6 \pm 1.3$ & $-0.4 \pm 0.9$ & $<0.001^{*}$ \\
\hline \multicolumn{7}{|l|}{ Secondary outcome variables } \\
\hline $\begin{array}{l}\text { \% cardiovascular risk } \\
\text { (UKPDS-OM2) }\end{array}$ & $-1.5 \pm 4$ & $-0.7 \pm 3.0$ & 0.49 & $-4.4 \pm 4.9$ & $-1.7 \pm 5.9$ & $0.008^{*}$ \\
\hline Diabetes empowerment & $1.3 \pm 5.0$ & $0.9 \pm 4.5$ & 0.84 & $3.8 \pm 4.5$ & $2.6 \pm 6.1$ & 0.22 \\
\hline Diabetes distress (emotional) & $0.0 \pm 0.7$ & $-0.2 \pm 0.9$ & 0.44 & $-0.4 \pm 1.0$ & $0.0 \pm 0.9$ & $0.015^{\star}$ \\
\hline Diabetes distress (regimen) & $-0.2 \pm 1.0$ & $0.5 \pm 0.6$ & 0.35 & $-1.0 \pm 1.4$ & $-0.2 \pm 1.0$ & $0.001^{*}$ \\
\hline $\begin{array}{l}\text { Depression (Patient Health } \\
\text { Questionnaire-9) }\end{array}$ & $0.8 \pm 4.0$ & $-1.6 \pm 2.8$ & 0.06 & $-1.0 \pm 4.0$ & $-0.6 \pm 5.3$ & 0.68 \\
\hline \multicolumn{7}{|l|}{ Side effect variables } \\
\hline $\mathrm{LDL}(\mathrm{mmol} / \mathrm{L})$ & $-0.0203 \pm 0.4871$ & $-0.1158 \pm 0.457$ & 0.56 & $-0.0288 \pm 0.5957$ & $0.0751 \pm 0.5367$ & 0.34 \\
\hline $\mathrm{HDL}(\mathrm{mmol} / \mathrm{L})$ & $0.0776 \pm 0.144$ & $-0.0111 \pm 0.1503$ & 0.09 & $0.1077 \pm 0.1897$ & $0.0259 \pm 0.1292$ & $0.010^{*}$ \\
\hline Triglycerides (mmol/L) & $-0.4064 \pm 1.1028$ & $0.0145 \pm 0.4735$ & 0.18 & $-0.3278 \pm 0.67$ & $-0.3914 \pm 1.9167$ & 0.81 \\
\hline Total cholesterol (mmol/L) & $-0.0103 \pm 0.5156$ & $-0.1194 \pm 0.4748$ & 0.52 & $-0.0538 \pm 0.7889$ & $-0.02 \pm 0.8357$ & 0.82 \\
\hline \multicolumn{7}{|l|}{ Mechanism variables } \\
\hline Diabetes knowledge & $0.1 \pm 3.3$ & $0.4 \pm 2.5$ & 0.80 & $3.1 \pm 2.7$ & $2.2 \pm 2.5$ & 0.06 \\
\hline Body mass index (BMI) & $-0.9 \pm 1.2$ & $-0.4 \pm 1.1$ & 0.20 & $-1.8 \pm 1.4$ & $-0.7 \pm 1.2$ & $<0.001^{*}$ \\
\hline $\begin{array}{l}\text { Carbohydrates Routinely } \\
\text { Consumed (servings) }\end{array}$ & $-1.6 \pm 14.2$ & $-5.1 \pm 16.3$ & 0.54 & $-21.0 \pm 17.0$ & $-12.0 \pm 20.0$ & $0.012^{*}$ \\
\hline SMBG readings (30 days) & $5.5 \pm 26.2$ & $1.7 \pm 28.7$ & 0.69 & $24.3 \pm 32.7$ & $10.8 \pm 30.8$ & $0.022^{*}$ \\
\hline $\begin{array}{l}\text { Glucose Monitor Satisfaction } \\
\text { Survey (GMSS) }\end{array}$ & $0.3 \pm 0.6$ & $0.3 \pm 0.7$ & 0.99 & $0.5 \pm 0.6$ & $0.2 \pm 0.5$ & $0.009^{*}$ \\
\hline $\begin{array}{l}\text { Medication Effect Score } \\
\text { (MES) }\end{array}$ & $-0.1 \pm 0.2$ & $0.3 \pm 0.6$ & 0.04 & $-0.1 \pm 0.5$ & $0.1 \pm 0.3$ & $0.015^{\star}$ \\
\hline
\end{tabular}

*Significant with a false discovery rate of $0.05 .^{16}$

GEM, glycemic excursion minimization; HDL, high-density lipoprotein; LDL, low-density lipoprotein; SMBG, self-monitoring of blood glucose; UKPDS-OM2, UK Prospective Diabetes Study Outcomes Model 2; WL, weight loss.

a significantly greater reduction of HbAlc and cardiovascular risk. Compared with GEM non-responders, GEM responders demonstrated additional benefits regarding less carbohydrates consumed, diabetes medication taken and diabetes distress, and greater satisfaction with BG monitoring, HDL, BMI, and diabetes empowerment. In conclusion, from the available data, GEM is equivalent

Table 5 Logistic regression comparing GEM responders to non-responders

\begin{tabular}{llll}
\hline Variables, GEM pre to post $\boldsymbol{\Delta}$ & OR & $\mathbf{9 5 \%} \mathbf{C l}$ & P value \\
\hline HbA1c & 2.02 & 1.32 to 3.08 & 0.001 \\
\hline Body mass index (BMI) & 1.58 & 1.11 to 2.27 & 0.012 \\
Medication Effect Score (MES) & 7.04 & 1.90 to 26.1 & 0.003 \\
\hline
\end{tabular}

GEM, glycemic excursion minimization. or superior to $\mathrm{WL}$ as a treatment option for the management of T2D.

Given the available data, responders did not differ from non-responders for either intervention when considering baseline demographics, psychological or disease severity variables. This indicates the broad applicability of both interventions.

While WL responders could not be predicted, $73 \%$ of GEM long-term responders were predicted by short-term improvement in reductions of $\mathrm{HbAlc}$, diabetes medication and BMI. While there may be some yet assessed physiological factors that contribute to being a GEM responder, our data suggest that for GEM participants only, the greater investment in making short-term goals may translate into more sustained benefits.

Theoretically, non-significant WL benefits may be partly due to lack of statistical power because of its 
smaller sample size. However, considering the WL p value levels in table 1 , power would probably only be relevant to diabetes distress (regimen), $\mathrm{p}=0.03$. Additionally, the limited efficacy of WL may have in part been due to its brevity, only six (once a week) face-to-face group sessions. This is in contrast to what the CDC recommends for a WL program for the prevention of diabetes, a yearlong program, ${ }^{19}$ or the 44 sessions employed by Look AHEAD in their initial year of treatment. ${ }^{20}$ However, to match groups in terms of therapist contact, this duration was presumed necessary. Generalization of these findings is limited by the above single-site recruitment of participants and a subject sample of predominantly white, college-educated adults. Furthermore, our past GEM interventions relied on face-to-face visits. Many clinics do not have the space or personnel to deliver such interventions and patients' routines may not permit such visits. A self-administered version would have significantly greater scalability.

GEM's current benefits are notable when considering that these outcomes are similar to those of more intensive WL lifestyle interventions that incorporated booster programs, ${ }^{20}$ and low or very low carbohydrate diets. ${ }^{21}$ GEM targets reducing immediate postnutrient BG excursions whereas ketogenic diets restrict total daily carbohydrate intake in order to induce a state of nutritional ketosis. GEM is not prescriptive targeting a certain number of calories or grams of carbohydrates but is personalized allowing patients to identify food and activity choices that positively or negatively impact their BG excursions.

Being a long-term GEM responder was associated with short-term improvements in HbAlc, cardiovascular risk, diabetes distress (emotional and regimen), HDL, BMI, carbohydrate ingestion, SMBG frequency and satisfaction, and MES. This suggests that being a long-term responder was related to being a short-term responder for GEM but not WL participants. While being a longterm GEM responder was associated with both short-term reduction of carbohydrates routinely consumed and reduction in weight, and reduction in carbohydrates did not account for a significant unique amount of variance in predicting responders, these analyses do not clarify the mechanisms underlying GEM sustainability.

The TTE contributed little in the comparison between GEM and WL responders. However, it did show a greater difference between responders and non-responders in the GEM intervention, as responders decreased their diabetes medication slightly and the non-responders increased their diabetes medications. The role of the TTE may become more important the longer the follow-up. This difference was not significant for the $\mathrm{WL}$ intervention.

The findings suggest that the paradigm shift in lifestyle intervention represented by GEM is a brief, broadly applicable, effective and durable treatment option for adults with T2D. These findings may be especially relevant to primary care providers who manage $\sim 90 \%$ of T2D. ${ }^{22}$ Implementation of the GEM intervention into primary care practices is likely to be feasible in current, real-world practices with existing care models. Delivery of GEM sessions could occur in diabetes group visits, and although delivered by certified diabetes education and care specialists in the study, GEM could also be delivered by other primary care practice staff or peer counselors.

Acknowledgements The authors are greatly indebted to their professional advisory board members, Dr Barbara Anderson, Dr Mary DeGroot, and Dr Lawrence Fisher, for their advice on the GEM manual, study design, data interpretation, grant applications, meeting abstracts and manuscripts. We are also indebted to Dr David Repaske for serving as our Human Subject Safety Monitor. We thank Victoria Ngo and Simone Buckman, who managed and assessed the participants, and our research assistants, Emily Klein, Camilla Schanche-Perret Gentil, and Brandon Smith, who collected and entered the data.

Contributors DJC oversaw the execution of the project, wrote the manuscript, and is the guarantor of the work. TO edited the manuscript and provided primary care perspective. MC oversaw and performed the data analyses. MM managed and analyzed the data. AM was the endocrinology consultant involved in the study design, oversaw all medical issues, participated in weekly team meetings and edited the manuscript.

Funding A grant from the NIH/NIDDK (5R01DK108957-02) funded the study. A grant from Dexcom (IIS-2017-047) provided additional equipment support.

Competing interests None declared.

\section{Patient consent for publication Not required.}

Ethics approval This study was performed according to the principles of the Declaration of Helsinki. Ethical approval for this study was obtained from the University of Virginia Institutional Review Board for Health Sciences Research (protocol number 19370).

Provenance and peer review Not commissioned; externally peer reviewed.

Data availability statement Data are available upon reasonable request. Please email the corresponding author regarding any data requests.

Open access This is an open access article distributed in accordance with the Creative Commons Attribution Non Commercial (CC BY-NC 4.0) license, which permits others to distribute, remix, adapt, build upon this work non-commercially, and license their derivative works on different terms, provided the original work is properly cited, appropriate credit is given, any changes made indicated, and the use is non-commercial. See: http://creativecommons.org/licenses/by-nc/4.0/.

ORCID iD

Daniel J Cox http://orcid.org/0000-0002-0910-5306

\section{REFERENCES}

1 Daousi C, Casson IF, Gill GV, et al. Prevalence of obesity in type 2 diabetes in secondary care: association with cardiovascular risk factors. Postgrad Med J 2006;82:280-4.

2 Penn L, White M, Lindström J, et al. Importance of weight loss maintenance and risk prediction in the prevention of type 2 diabetes: analysis of European diabetes prevention study RCT. PLoS One 2013;8:e57143.

3 Ketema EB, Kibret KT. Correlation of fasting and postprandial plasma glucose with $\mathrm{HbA} 1 \mathrm{c}$ in assessing glycemic control; systematic review and meta-analysis. Arch Public Health 2015;73:1-9.

4 Ceriello A, Davidson J, Hanefeld M, et al. Postprandial hyperglycaemia and cardiovascular complications of diabetes: an update. Nutrit, Metab Card Dis 2006;16:453-6.

5 Cox DJ, Banton T, Moncrief M, et al. Minimizing glucose excursions (GEM) with continuous glucose monitoring in type 2 diabetes: a randomized clinical trial. J Endocr Soc 2020;4:bvaa118.

6 Cox DJ, Taylor AG, Singh H, et al. Glycemic load, exercise, and monitoring blood glucose (GEM): a paradigm shift in the treatment of type 2 diabetes mellitus. Diabetes Res Clin Pract 2016;111:28-35.

7 Cox DJ, Banton T, Moncrief M. Glycemic excursion minimization in the management of type 2 diabetes: a novel intervention tested in a randomized clinical trial. BMJ Open Diabetes Res Care.

8 Cox DJ, Taylor AG, Moncrief M, et al. Continuous glucose monitoring in the self-management of type 2 diabetes: a paradigm shift. Diabetes Care 2016;39:e71-3. 
9 Hayes AJ, Leal J, Gray AM, et al. UKPDS outcomes model 2: a new version of a model to simulate lifetime health outcomes of patients with type 2 diabetes mellitus using data from the 30 year United Kingdom prospective diabetes study: UKPDS 82. Diabetologia 2013;56:1925-33.

10 Polonsky WH, Fisher L, Earles J, et al. Assessing psychosocial distress in diabetes: development of the diabetes distress scale. Diabetes Care 2005;28:626-31.

11 Anderson RM, Funnell MM, Fitzgerald JT, et al. The diabetes empowerment scale: a measure of psychosocial self-efficacy. Diabetes Care 2000;23:739-43.

12 Kroenke K, Spitzer RL, Williams JB. The PHQ-9: validity of a brief depression severity measure. J Gen Intern Med 2001;16:606-13.

13 Polonsky WH, Fisher L, Hessler D, et al. Development of a new measure for assessing glucose monitoring device-related treatment satisfaction and quality of life. Diabetes Technol Ther 2015;17:657-63.

14 Alexopoulos A-S, Yancy WS, Edelman D, et al. Clinical associations of an updated medication effect score for measuring diabetes treatment intensity. Chronic IIIn 2021;17:451-62.

15 Centers for Disease Control \& Prevention, National Diabetes Prevention Program. Tools and resources: curricula and handouts. Available: https://www.cdc.gov/diabetes/prevention/resources/
curriculum.html?CDC_AA_refVal=https $\% 3 A \% 2 F \% 2 F w w w$. cdc.gov\%2Fdiabetes\%2Fprevention\%2Flifestyle-program\% 2Fcurriculum.html [Accessed 10 Dec 2020].

16 Benjamini Y, Hochberg Y. Controlling the false discovery rate: a practical and powerful approach to multiple testing. $J R$ Stat Soc Series B Stat Methodol 1995;57:289-300.

17 Anderson RM, Funnell MM. The art of empowerment: psychology in diabetes care. Am Diabet Assoc 2000.

18 Hernandez-Tejada MA, Campbell JA, Walker RJ, et al. Diabetes empowerment, medication adherence and self-care behaviors in adults with type 2 diabetes. Diabetes Technol Ther 2012;14:630-4.

19 CDC. National diabetes prevention program. Diabetes.

20 Look AHEAD Research Group, Wing RR. Long-term effects of a lifestyle intervention on weight and cardiovascular risk factors in individuals with type 2 diabetes mellitus: four-year results of the look AHEAD trial. Arch Intern Med 2010;170:1566-75.

21 Goldenberg JZ, Day A, Brinkworth GD, et al. Efficacy and safety of low and very low carbohydrate diets for type 2 diabetes remission: systematic review and meta-analysis of published and unpublished randomized trial data. BMJ 2021;372:m4743.

22 Unger J, Kushner P, Anderson JE. Practical guidance for using the FreeStyle Libre flash continuous glucose monitoring in primary care. Postgrad Med 2020;132:305-13. 